\title{
On Improved Delay-dependent Robust Stability Criteria for Uncertain Systems with Interval Time-varying Delay
}

\author{
Jun-Jun Hui ${ }^{1,2} \quad$ He-Xin Zhang ${ }^{1} \quad$ Xiang-Yu Kong ${ }^{1} \quad$ Xin Zhou ${ }^{1}$ \\ ${ }^{1}$ The Xi'an Research Institute of High Technology, Xi'an 710025, China \\ ${ }^{2}$ The Baoji Research Institute of High Technology, Baoji 721013, China
}

\begin{abstract}
This paper considers the problem of delay-dependent robust stability for uncertain systems with interval time-varying delays. By using the direct Lyapunov method, a new Lyapunov-Krasovskii (L-K) functional is introduced based on decomposition approach, when dealing with the time derivative of L-K functional, a new tight integral inequality is adopted for bounding the cross terms. Then, a new less conservative delay-dependent stability criterion is formulated in terms of linear matrix inequalities (LMIs), which can be easily solved by optimization algorithms. Numerical examples are given to show the effectiveness and the benefits of the proposed method.
\end{abstract}

Keywords: Lyapunov-Krasovskii (L-K) functional, delay decomposition approach, linear matrix inequality (LMI), interval timevarying delay, robust stability.

\section{Introduction}

Time-delay phenomena are ubiquitous in many practical systems such as chemical engineering systems, biological systems, economic systems and networked control systems, which are often major sources of instability and poor performance. Hence, stability analysis and stabilization of systems with time-delays have received considerable attention in the past few years (see e.g. [1-24], and the references therein). Very recently, systems with time-varying delay in a known interval have been studied in [3, 7-15, 20-22], wherein the time-delay may vary in a range for which the lower bound is not restricted to being zero.

The existing stability criteria are usually classified into the delay-independent ones and the delay-dependent ones. Since the delay-dependent ones are generally less conservative than the delay-independent ones, especially for small delays, much attention has been paid to the delaydependent ones. An important issue in this field is to enlarge the feasible region of stability criteria. For this purpose, many approaches have been developed in the past few years, among which the free-weighting matrix method and Jensen's integral inequality method are widely used and obtained plentiful results (see e.g. [2-14], and the references therein). For the purpose of reducing the conservatism caused by the use of system transformations and bounding techniques, the free-weighting matrix method was first proposed in $[2,3]$ to investigate the stability of systems with time-varying delay. By employing a convex combination

\footnotetext{
Brief paper

Manuscript received August 10, 2013; accepted January 27, 2014

This work was supported by National Natural Science Foundation of China (No. 61074072).

Recommended by Associate Editor Min Wu

(C)Institute of Automation, Chinese Academy of Science and Springer-Verlag Berlin Heidelberg 2015
}

technique, Park and $\mathrm{Ko}^{[4]}$ further extended the free weighting matrix method to a new Lyapunov functional. Recently, motivated by the idea of L-K functional with tripleintegral terms, an extended free-weighting matrix method, i.e., double-integral inequality method was proposed in [5]. Although the free-weighting matrix method can effectively reduce the conservatism, it can also lead to the increase of computational complexity since many slack variables are introduced.

Jensen's integral inequality approach is another important one, which was first introduced by $\mathrm{Gu}^{[6]}$ for stability analysis of time-delay systems. Soon afterwards, many researchers, such as $\operatorname{Han}^{[7]}$, Zhang and Han ${ }^{[8]}$, Sun et al. ${ }^{[9]}$, Kwon et al. ${ }^{[10]}$, Ramakrishnan and Ray ${ }^{[11]}$ further extended the Jensen's integral inequality to some new forms. Jensen's integral inequality approach can provide a simple form of stability conditions without introducing redundant variables. However, how to construct a less conservative inequality is a difficult problem. Recently, a novel technique called delay-central point method was proposed in [16], by employing a central point of variation about the delay range, the time-interval is divided into two segments of equal length, and the time variation of a candidate L-K functional is evaluated individually in each subinterval. In order to further reduce the conservatism, the delay decomposition approach was proposed in [17-20], and show its improvement of maximum delay bounds. Based on decomposition technique, Ramakrishnan and Ray ${ }^{[21,22]}$ further extended the delay-central point method and proposed a less conservative stability criteria. Nevertheless, there still exists room for further improvements.

Motivated by the above discussions, we further discuss the stability of uncertain linear systems with interval timevarying delays. Based on the direct L-K approach and linear 
matrix inequality, new less conservative delay-dependent stability criteria for computing the maximum allowable bound of the delay range is formulated in terms of linear matrix inequalities. Less conservative results are mainly attributed to the decomposition technique for designing the L-K functional and tighter bounding technique for dealing with the cross-terms. Numerical examples are given to illustrate the effectiveness and less conservatism of the proposed method.

Notations. Throughout this paper, $\mathbf{R}^{n}$ denotes the $n$ dimensional Euclidian space, $\mathbf{R}^{n \times m}$ is the set of $n \times m$ real matrices, the notation $X>0$, for $X \in \mathbf{R}^{n \times m}$ means that the matrix $X$ is a real symmetric positive definite. For an arbitrary matrix $B$ and two symmetric matrices $A$ and $C$, $\left[\begin{array}{ll}A & B \\ * & C\end{array}\right]$ denotes a symmetric matrix, where $*$ denotes the entries implied by symmetry.

\section{Problem description and preliminar- ies}

Consider the following time-varying delay system:

$$
\left\{\begin{array}{l}
\dot{x}(t)=(A+\Delta A(t)) x(t)+(B+\Delta B(t)) x(t-h(t)) \\
x(t)=\varphi(t), \quad t \in\left[-h_{2}, 0\right]
\end{array}\right.
$$

where $x(t) \in \mathbf{R}^{n}$ is the state vector, $A$ and $B$ are constant matrices with appropriate dimensions, $h(t)$ is a timevarying delay satisfying the following two categories:

$$
\begin{aligned}
& \text { Case } 1: 0 \leq h_{1} \leq h(t) \leq h_{2}, \dot{h}(t) \leq \mu, \forall t \geq 0 \\
& \text { Case } 2: 0 \leq h_{1} \leq h(t) \leq h_{2}, \forall t \geq 0
\end{aligned}
$$

$\Delta A(t)$ and $\Delta B(t)$ denote the parameter uncertainties satisfying the following condition:

$$
\left[\begin{array}{ll}
\Delta A(t) & \Delta B(t)
\end{array}\right]=D F(t)\left[\begin{array}{ll}
E_{a} & E_{b}
\end{array}\right]
$$

where $D, E_{a}$ and $E_{b}$ are constant matrices with appropriate dimensions, and $F(t)$ is an unknown time-varying matrix, which is Lesbesgue, satisfying, $F(t)^{\mathrm{T}} F(t) \leq I$.

Before moving on, the following lemma is necessary in the proof of the main results.

Lemma 1. For any scalar $h(t) \geq 0$, and any constant matrix $Q \in \mathbf{R}^{n \times n}, Q=Q^{\mathrm{T}}>0$, the following inequality holds:

$$
\begin{gathered}
-\int_{t-h(t)}^{t} \dot{x}^{\mathrm{T}}(s) Q \dot{x}(s) \mathrm{d} s \leq h(t) \zeta^{\mathrm{T}}(t) V Q^{-1} V^{\mathrm{T}} \zeta(t)+ \\
2 \zeta^{\mathrm{T}}(t) V[x(t)-x(t-h(t))]
\end{gathered}
$$

where

$$
\begin{aligned}
& \zeta^{\mathrm{T}}(t)=\left[\begin{array}{llll}
x^{\mathrm{T}}(t) & x^{\mathrm{T}}\left(t-h_{1}\right) & \Gamma_{a}^{\mathrm{T}}(t) & x^{\mathrm{T}}(t-h(t))
\end{array}\right. \\
& \left.\Gamma_{2}^{\mathrm{T}}(t) \quad \dot{x}^{\mathrm{T}}(t)\right] \\
& \Gamma_{a}^{\mathrm{T}}(t)=\left[x^{\mathrm{T}}\left(t-\frac{h_{a}}{N}\right) \quad x^{\mathrm{T}}\left(t-2 \frac{h_{a}}{N}\right) \quad \cdots\right. \\
& \left.x^{\mathrm{T}}\left(t-(N-1) \frac{h_{a}}{N}\right) \quad x^{\mathrm{T}}\left(t-h_{a}\right)\right] \\
& \Gamma_{2}^{\mathrm{T}}(t)=\left[x^{\mathrm{T}}\left(t-\frac{h_{2}}{N}\right) \quad x^{\mathrm{T}}\left(t-2 \frac{h_{2}}{N}\right) \quad \cdots\right. \\
& \left.x^{\mathrm{T}}\left(t-(N-1) \frac{h_{2}}{N}\right) \quad x^{\mathrm{T}}\left(t-h_{2}\right)\right]
\end{aligned}
$$

in which $h_{a}=\frac{\left(h_{1}+h_{2}\right)}{2}, V$ is free weighting matrix with appropriate dimensions, and $N$ is a given positive integer.

Proof. For any real vectors $a, b$ and any matrix $Q>0$ with appropriate dimensions, we know that the following inequality holds

$$
\pm 2 a^{\mathrm{T}} b \leq a^{\mathrm{T}} Q a+b^{\mathrm{T}} Q^{-1} b .
$$

From (5), we obtain

$$
\begin{aligned}
& -2 \int_{t-h(t)}^{t}\left(V^{\mathrm{T}} \zeta(t)\right)^{\mathrm{T}} \dot{x}(s) \mathrm{d} s \leq \int_{t-h(t)}^{t} \dot{x}^{\mathrm{T}}(s) Q \dot{x}(s) \mathrm{d} s+ \\
& \int_{t-h(t)}^{t} \zeta^{\mathrm{T}}(t) V Q^{-1} V^{\mathrm{T}} \zeta(t) \mathrm{d} s .
\end{aligned}
$$

So we have

$$
\begin{aligned}
& -\int_{t-h(t)}^{t} \dot{x}^{\mathrm{T}}(s) Q \dot{x}(s) \mathrm{d} s \leq 2 \int_{t-h(t)}^{t}\left(V^{\mathrm{T}} \zeta(t)\right)^{\mathrm{T}} \dot{x}(s) \mathrm{d} s+ \\
& \int_{t-h(t)}^{t} \zeta^{\mathrm{T}}(t) V Q^{-1} V^{\mathrm{T}} \zeta(t) \mathrm{d} s= \\
& h(t) \zeta^{\mathrm{T}}(t) V Q^{-1} V^{\mathrm{T}} \zeta(t)+2 \zeta^{\mathrm{T}}(t) V[x(t)-x(t-h(t))] .
\end{aligned}
$$

Lemma $\mathbf{2}^{[25]}$. Suppose $\gamma_{1} \leq \gamma(t) \leq \gamma_{2}$, where $\gamma(\cdot)$ : $\mathbf{R}_{+} \rightarrow \mathbf{R}_{+}$. Then, for any constant matrices $\Xi_{1}, \Xi_{2}$ and $\Omega$ with proper dimensions, the following matrix inequality

$$
\Omega+\left(\gamma(t)-\gamma_{1}\right) \Xi_{1}+\left(\gamma_{2}-\gamma(t)\right) \Xi_{2}<0
$$

holds, if and only if

$$
\Omega+\left(\gamma_{2}-\gamma_{1}\right) \Xi_{1}<0, \quad \Omega+\left(\gamma_{2}-\gamma_{1}\right) \Xi_{2}<0 .
$$

Lemma $3^{[26]}$. Given matrices $Q=Q^{\mathrm{T}}, H, E$ and $R=R^{\mathrm{T}}$ with appropriate dimensions, the inequality

$$
Q+H F E+E^{\mathrm{T}} F^{\mathrm{T}} H^{\mathrm{T}}<0
$$

for all $F$ satisfying $F^{\mathrm{T}} F \leq R$, if and only if there exists some scalar $\varepsilon>0$, such that

$$
Q+\varepsilon H H^{\mathrm{T}}+\varepsilon^{-1} E^{\mathrm{T}} R E<0 .
$$




\section{Main results}

In this section, we shall establish our main results based on LMI framework. First, we will consider nominal system, whereafter, we study the robust stability of the uncertain system. Consider the nominal system of (1):

$$
\left\{\begin{array}{l}
\dot{x}(t)=A x(t)+B x(t-h(t)) \\
x(t)=\varphi(t), t \in\left[-h_{2}, 0\right] .
\end{array}\right.
$$

Theorem 1. For given values of $h_{1}, h_{2}$ and $\mu$, system (6) is asymptotically stable, if there exist real symmetric positive definitive matrices $P_{1}, P_{2}, P_{3}, Z_{1}, Z_{2}$; symmetric matrices $Q, S$ and free matrix variables $L_{j}, M_{j}, V_{j}, T_{j}$ $(j=1,2)$ with appropriate dimensions such that the following LMIs hold:

$$
\begin{aligned}
& {\left[\begin{array}{ccc}
\Phi & \sqrt{\frac{h_{a}}{N}} L & \sqrt{h_{\delta}} M \\
* & -Z_{1} & 0 \\
* & * & -Z_{2}
\end{array}\right]<0} \\
& {\left[\begin{array}{ccc}
\Phi & \sqrt{\frac{h_{a}}{N}} L & \sqrt{h_{\delta}} V \\
* & -Z_{1} & 0 \\
* & * & -Z_{2}
\end{array}\right]<0}
\end{aligned}
$$

and

$$
\begin{aligned}
Q & =\left[\begin{array}{cccc}
Q_{11} & Q_{12} & \ldots & Q_{1 N} \\
* & Q_{22} & \ldots & Q_{2 N} \\
\vdots & \vdots & \ddots & \vdots \\
* & * & Q_{N-1, N-1} & Q_{N-1, N} \\
* & * & * & Q_{N N}
\end{array}\right] \geq 0 \\
S & =\left[\begin{array}{cccc}
S_{11} & S_{12} & \ldots & S_{1 N} \\
* & S_{22} & \ldots & S_{2 N} \\
\vdots & \vdots & \ddots & \vdots \\
* & * & S_{N-1, N-1} & S_{N-1, N} \\
* & * & * & S_{N N}
\end{array}\right] \geq 0
\end{aligned}
$$

where

$$
\Phi=\left[\begin{array}{cccc}
\Phi_{11} & \Phi_{12} & \Phi_{13} & \Phi_{14} \\
* & \Phi_{22} & \Phi_{23} & B^{\mathrm{T}} T_{2}^{\mathrm{T}} \\
* & * & \Phi_{33} & \mathbf{0} \\
* & * & * & \Phi_{44}
\end{array}\right]
$$

$\Phi_{11}=$

$$
\left[\begin{array}{ccccccc}
\Phi_{1} & 0 & \Phi_{2} & Q_{13} & \cdots & Q_{1 N} & 0 \\
* & P_{3}-P_{2} & 0 & 0 & \cdots & 0 & 0 \\
* & * & \Phi_{3} & Q_{23}-Q_{12} & \cdots & Q_{2 N}-Q_{1, N-1} & -Q_{1 N} \\
* & * & * & Q_{33}-Q_{22} & \cdots & Q_{3 N}-Q_{2, N-1} & -Q_{2 N} \\
\vdots & \vdots & \vdots & \vdots & \ddots & \vdots & \vdots \\
* & * & * & * & * & Q_{N N}-Q_{N-1, N-1} & -Q_{N-1, N} \\
* & * & * & * & * & * & V_{1}+V_{1}^{\mathrm{T}}-Q_{N, N}
\end{array}\right]
$$

$$
\begin{aligned}
& \Phi_{33}= \\
& {\left[\begin{array}{ccccc}
S_{22}-S_{11} & S_{23}-S_{12} & \cdots & S_{2 N}-S_{1, N-1} & -S_{1 N} \\
* & S_{33}-S_{22} & \cdots & S_{3 N}-S_{2, N-1} & -S_{2 N} \\
& & \ddots & \vdots & \vdots \\
* & * & \ddots & & \\
\vdots & \vdots & \cdots & S_{N N}-S_{N-1, N-1} & -S_{N-1, N} \\
* & * & * & * & -S_{N N}-M_{2}-M_{2}^{\mathrm{T}}
\end{array}\right]} \\
& \Phi_{13}=\left[\begin{array}{ccccc}
S_{12} & S_{13} & \cdots & S_{1 N} & 0 \\
0 & 0 & \cdots & 0 & 0 \\
0 & 0 & \cdots & 0 & 0 \\
\vdots & \vdots & \ddots & \vdots & \vdots \\
0 & 0 & \cdots & 0 & 0
\end{array}\right] \\
& \Phi_{1}=P_{2}+Q_{11}+S_{11}+L_{1}+L_{1}^{\mathrm{T}}+T_{1} A+A^{\mathrm{T}} T_{1}^{\mathrm{T}} \\
& \Phi_{2}=Q_{12}-L_{1}+L_{2}^{\mathrm{T}}, \quad \Phi_{3}=Q_{22}-Q_{11}-L_{2}-L_{2}^{\mathrm{T}} \\
& \Phi_{12}=\left[\begin{array}{lllll}
\left(T_{1} B\right)^{\mathrm{T}} & 0 & \cdots & 0 & \left(-V_{1}+V_{2}^{\mathrm{T}}\right)^{\mathrm{T}}
\end{array}\right]^{\mathrm{T}} \\
& \Phi_{14}=\left[\begin{array}{llll}
\left(P_{1}-T_{1}+A^{\mathrm{T}} T_{2}^{\mathrm{T}}\right)^{\mathrm{T}} & 0 & \cdots & 0
\end{array}\right]^{\mathrm{T}} \\
& \Phi_{22}=-(1-\mu) P_{3}-V_{2}-V_{2}^{\mathrm{T}}+M_{1}+M_{1}^{\mathrm{T}} \\
& \Phi_{23}=\left[\begin{array}{llll}
0 & \cdots & 0 & -M_{1}+M_{2}^{\mathrm{T}}
\end{array}\right] \\
& \Phi_{44}=\frac{h_{a}}{N} Z_{1}+h_{\delta} Z_{2}-T_{2}-T_{2}^{\mathrm{T}} \\
& h_{a}=\frac{\left(h_{1}+h_{2}\right)}{2}, \quad h_{\delta}=\frac{\left(h_{2}-h_{1}\right)}{2} \\
& L=\left[\begin{array}{llllllll}
L_{1}^{\mathrm{T}} & 0 & L_{2}^{\mathrm{T}} & 0 & 0 & \cdots & 0 & 0
\end{array}\right]^{\mathrm{T}} \\
& V=\left[\begin{array}{llllllll}
0 & \cdots & 0 & V_{1}^{\mathrm{T}} & V_{2}^{\mathrm{T}} & 0 & \cdots & 0
\end{array}\right]^{\mathrm{T}} \\
& T=\left[\begin{array}{cccccccc}
T_{1}^{\mathrm{T}} & 0 & 0 & 0 & \cdots & 0 & 0 & T_{2}^{\mathrm{T}}
\end{array}\right]^{\mathrm{T}} \\
& M=\left[\begin{array}{llllllll}
0 & \cdots & 0 & M_{1}^{\mathrm{T}} & 0 & \cdots & M_{2}^{\mathrm{T}} & 0
\end{array}\right]^{\mathrm{T}} .
\end{aligned}
$$

Proof. Construct an L-K functional candidate as

$$
V(t)=V_{1}(t)+V_{2}(t)+V_{3}(t)
$$

where

$$
\begin{aligned}
& V_{1}(t)=x^{\mathrm{T}}(t) P_{1} x(t)+\int_{t-h_{1}}^{t} x^{\mathrm{T}}(s) P_{2} x(s) \mathrm{d} s+ \\
& \int_{t-h(t)}^{t-h_{1}} x^{\mathrm{T}}(s) P_{3} x(s) \mathrm{d} s \\
& V_{2}(t)=\int_{t-\frac{h_{a}}{N}}^{t} \xi_{1}^{\mathrm{T}}(s) Q \xi_{1}(s) \mathrm{d} s+\int_{t-\frac{h_{2}}{N}}^{t} \xi_{2}^{\mathrm{T}}(s) S \xi_{2}(s) \mathrm{d} s \\
& V_{3}(t)=\int_{-\frac{h_{a}}{N}}^{0} \int_{t+\theta}^{t} \dot{x}^{\mathrm{T}}(s) Z_{1} \dot{x}(s) \mathrm{d} s \mathrm{~d} \theta+ \\
& \int_{-h_{2}}^{-h_{a}} \int_{t+\theta}^{t} \dot{x}^{\mathrm{T}}(s) Z_{2} \dot{x}(s) \mathrm{d} s \mathrm{~d} \theta
\end{aligned}
$$

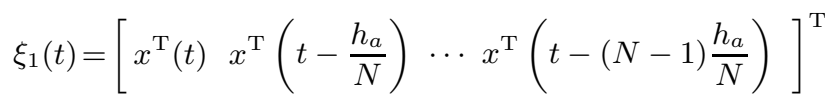

$$
\begin{aligned}
& \xi_{2}(t)=\left[\begin{array}{lll}
x^{\mathrm{T}}(t) & x^{\mathrm{T}}\left(t-\frac{h_{2}}{N}\right) \cdots x^{\mathrm{T}}\left(t-(N-1) \frac{h_{2}}{N}\right)
\end{array}\right]^{\mathrm{T}} .
\end{aligned}
$$

The time-derivative of the L-K functional along the tra- 
jectory of (6) is given by

$$
\dot{V}(t)=\dot{V}_{1}(t)+\dot{V}_{2}(t)+\dot{V}_{3}(t)
$$

where

$$
\begin{aligned}
\dot{V}_{1}(t) \leq & 2 x^{\mathrm{T}}(t) P_{1} \dot{x}(t)+x^{\mathrm{T}}(t) P_{2} x(t)+ \\
& x^{\mathrm{T}}\left(t-h_{1}\right)\left(P_{3}-P_{2}\right) x\left(t-h_{1}\right)- \\
& (1-\mu) x^{\mathrm{T}}(t-h(t)) P_{3} x(t-h(t)) \\
\dot{V}_{2}(t)= & \xi_{1}^{\mathrm{T}}(t) Q \xi_{1}(t)-\xi_{1}^{\mathrm{T}}\left(t-\frac{h_{a}}{N}\right) Q \xi_{1}\left(t-\frac{h_{a}}{N}\right)+ \\
& \xi_{2}^{\mathrm{T}}(t) S \xi_{2}(t)-\xi_{2}^{\mathrm{T}}\left(t-\frac{h_{2}}{N}\right) S \xi_{2}\left(t-\frac{h_{2}}{N}\right) \\
\dot{V}_{3}(t)= & \frac{h_{a}}{N} \dot{x}^{\mathrm{T}}(t) Z_{1} \dot{x}(t)+\left(h_{2}-h_{a}\right) \dot{x}^{\mathrm{T}}(t) Z_{2} \dot{x}(t)- \\
& \int_{t-\frac{h_{a}}{N}}^{t} \dot{x}^{\mathrm{T}}(s) Z_{1} \dot{x}(s) \mathrm{d} s-\int_{t-h_{2}}^{t-h_{a}} \dot{x}^{\mathrm{T}}(s) Z_{2} \dot{x}(s) \mathrm{d} s .
\end{aligned}
$$

Note that

$$
\begin{aligned}
-\int_{t-h_{2}}^{t-h_{a}} \dot{x}^{\mathrm{T}}(s) Z_{2} \dot{x}(s) \mathrm{d} s= & -\int_{t-h_{2}}^{t-h(t)} \dot{x}^{\mathrm{T}}(s) Z_{2} \dot{x}(s) \mathrm{d} s- \\
& \int_{t-h(t)}^{t-h_{a}} \dot{x}^{\mathrm{T}}(s) Z_{2} \dot{x}(s) \mathrm{d} s .
\end{aligned}
$$

Using Lemma 1, one can obtain

$$
\begin{aligned}
& -\int_{t-\frac{h_{a}}{N}}^{t} \dot{x}^{\mathrm{T}}(s) Z_{1} \dot{x}(s) \mathrm{d} s \leq \frac{h_{a}}{N} \zeta^{\mathrm{T}}(t) L Z_{1}^{-1} L^{\mathrm{T}} \zeta(t)+ \\
& 2 \zeta^{\mathrm{T}}(t) L\left[x(t)-x\left(t-\frac{h_{a}}{N}\right)\right] \\
& -\int_{t-h(t)}^{t-h_{a}} \dot{x}^{\mathrm{T}}(s) Z_{2} \dot{x}(s) \mathrm{d} s \leq\left(h(t)-h_{a}\right) \zeta^{\mathrm{T}}(t) V Z_{2}^{-1} V^{\mathrm{T}} \zeta(t)+ \\
& 2 \zeta^{\mathrm{T}}(t) V\left[x\left(t-h_{a}\right)-x(t-h(t))\right] \\
& -\int_{t-h_{2}}^{t-h(t)} \dot{x}^{\mathrm{T}}(s) Z_{2} \dot{x}(s) \mathrm{d} s \leq\left(h_{2}-h(t)\right) \zeta^{\mathrm{T}}(t) M Z_{2}^{-1} M^{\mathrm{T}} \zeta(t)+ \\
& 2 \zeta^{\mathrm{T}}(t) M\left[x(t-h(t))-x\left(t-h_{2}\right)\right] .
\end{aligned}
$$

On the other hand, for any matrices $T$ with appropriate dimensions, and from system (6), we have

$$
0=2 \zeta^{\mathrm{T}}(t) T[A x(t)+B x(t-h(t))-\dot{x}(t)]
$$

by substituting (11)-(14) in (10), the time derivative $\dot{V}(t)$ can be expressed as

$$
\dot{V}(t) \leq \zeta^{\mathrm{T}}(t) \Lambda \zeta(t)
$$

where

$$
\begin{aligned}
\Lambda= & \Phi+\frac{h_{a}}{N} L Z_{1}^{-1} L^{\mathrm{T}}+\left(h_{2}-h(t)\right) M Z_{2}^{-1} M^{\mathrm{T}}+ \\
& \left(h(t)-h_{a}\right) V Z_{2}^{-1} V^{\mathrm{T}} .
\end{aligned}
$$

If $\forall h(t) \in\left[h_{1}, h_{2}\right]$

$$
\Lambda<0 .
$$

Then $\dot{V}(t)<-\varepsilon\|x(t)\|^{2}$ for some scalar $\varepsilon>0$, from which we conclude that the nominal system (6) is asymptotically stable according to Lyapunov stability theory.
Now, we apply Lemma 2 to (16) to yield the following inequalities:

$$
\begin{aligned}
& \Phi+\frac{h_{a}}{N} L Z_{1}^{-1} L^{\mathrm{T}}+\left(h_{2}-h_{a}\right) M Z_{2}^{-1} M^{\mathrm{T}}<0 \\
& \Phi+\frac{h_{a}}{N} L Z_{1}^{-1} L^{\mathrm{T}}+\left(h_{2}-h_{a}\right) V Z_{2}^{-1} V^{\mathrm{T}}<0 .
\end{aligned}
$$

Schur complement on (17) and (18) yields the LMIs stated in the Theorem 1.

Remark 1. Different from the decomposition approach used in [22], when dealing with the time derivative of L-K functional, we proposed a new tight integral inequality (Lemma 1) for bounding the cross terms, hence, it yields a less conservative result. The comparisons of conservatism with some existing methods will be presented in Section 4

Remark 2. When the information of the time derivative $h(t)$ is unknown, by choosing $P_{3}=0$, we can get delaydependent and rate-independent stability criterion from Theorem 1.

Next, we study the robust stability of the uncertain system (1).

Theorem 2. For given values of $h_{1}, h_{2}$ and $\mu$, system (1) is asymptotically stable, if there exist a scalar $\varepsilon_{i}>0(i=1,2)$, and real symmetric positive definitive matrices $P_{1}, P_{2}, P_{3}, Z_{1}, Z_{2}$, symmetric matrices $Q, S$ and free matrix variables $L_{j}, M_{j}, V_{j}, T_{j}(j=1,2)$, with appropriate dimensions such that the following LMIs hold:

$$
\begin{aligned}
& {\left[\begin{array}{ccc}
\hat{\Phi}_{1} & \Gamma_{1}^{\mathrm{T}} D & \varepsilon_{1} \Gamma_{2}^{\mathrm{T}} \\
* & -\varepsilon_{1} I & 0 \\
* & * & -\varepsilon_{1} I
\end{array}\right]<0} \\
& {\left[\begin{array}{ccc}
\hat{\Phi}_{2} & \Gamma_{1}^{\mathrm{T}} D & \varepsilon_{2} \Gamma_{2}^{\mathrm{T}} \\
* & -\varepsilon_{2} I & 0 \\
* & * & -\varepsilon_{2} I
\end{array}\right]<0}
\end{aligned}
$$

and

$$
\begin{aligned}
Q & =\left[\begin{array}{cccc}
Q_{11} & Q_{12} & \ldots & Q_{1 N} \\
* & Q_{22} & \ldots & Q_{2 N} \\
\vdots & \vdots & \ddots & \vdots \\
* & * & Q_{N-1, N-1} & Q_{N-1, N} \\
* & * & * & Q_{N N}
\end{array}\right] \geq 0 \\
S & =\left[\begin{array}{cccc}
S_{11} & S_{12} & \ldots & S_{1 N} \\
* & S_{22} & \ldots & S_{2 N} \\
\vdots & \vdots & \ddots & \vdots \\
* & * & S_{N-1, N-1} & S_{N-1, N} \\
* & * & * & S_{N N}
\end{array}\right] \geq 0
\end{aligned}
$$


where

$$
\begin{aligned}
& \hat{\Phi}_{1}=\left[\begin{array}{ccc}
\Phi & \sqrt{\frac{h_{a}}{N}} L & \sqrt{h_{\delta}} M \\
* & -Z_{1} & 0 \\
* & * & -Z_{2}
\end{array}\right] \\
& \hat{\Phi}_{2}=\left[\begin{array}{ccc}
\Phi & \sqrt{\frac{h_{a}}{N}} L & \sqrt{h_{\delta}} V \\
* & -Z_{1} & 0 \\
* & * & -Z_{2}
\end{array}\right] \\
& \Gamma_{1}=\left[\begin{array}{lllllll}
T_{1}^{\mathrm{T}} & 0 & \cdots & 0 & T_{2}^{\mathrm{T}} & 0 & 0
\end{array}\right] \\
& \Gamma_{2}=\left[\begin{array}{llllllll}
E_{a} & 0 & \cdots & 0 & E_{b} & 0 & \cdots & 0
\end{array}\right] \text {. }
\end{aligned}
$$

Proof. Replacing $A$ and $B$ in Theorem 1 with $A+\Delta A$, $B+\Delta B$, respectively, and using Lemma 3 completes the proof.

\section{Numerical examples}

In this section, three numerical examples are given to show that the proposed approach reduces the conservativeness compared with some of the existing ones.
Example $\mathbf{1}^{[3]}$. Consider a nominal time-delay system with the following parameters:

$$
A=\left[\begin{array}{cc}
-2 & 0 \\
0 & -0.9
\end{array}\right], \quad B=\left[\begin{array}{cc}
-1 & 0 \\
-1 & -1
\end{array}\right] .
$$

For given $\mu$ and unknown $\mu$, Tables 1 and 2 provides the maximal allowable bounds of the delay $h_{2}$ for given lower bounds $h_{1}$, respectively. From the tables, it is clear that the proposed stability criterion is less conservative than those in $[3,14,16,19-22]$ even when we use small delay partitioning number. Furthermore, with the increase of the delay partitioning number, the conservatism will gradually reduce. The less conservative results are mainly attributed to the use of the tighter integral inequalities (Lemma 1) for dealing with the cross-terms.

Example 2 ${ }^{[13]}$. Consider an uncertain system described $A=\left[\begin{array}{cc}-2+\delta_{1} & 0 \\ 0 & -1+\delta_{2}\end{array}\right], \quad B=\left[\begin{array}{cc}-1+\delta_{3} & 0 \\ -1 & -1+\delta_{4}\end{array}\right]$

\begin{tabular}{|c|c|c|c|c|c|c|c|}
\hline$\mu$ & Method & $h_{1}=0$ & $h_{1}=1$ & $h_{1}=2$ & $h_{1}=3$ & $h_{1}=4$ & $h_{1}=5$ \\
\hline \multirow{8}{*}{0.5} & {$[3]$} & 2.0439 & 2.0764 & 2.4328 & 3.2234 & 4.0643 & - \\
\hline & {$[14]$} & 2.0723 & 2.1276 & 2.5048 & 3.2591 & 4.0744 & - \\
\hline & {$[16]$} & 2.0801 & 2.1513 & 2.7113 & 3.3839 & 4.1136 & - \\
\hline & {$[21]$} & 2.1484 & 2.3239 & 2.8630 & 3.5729 & 4.3343 & 5.1306 \\
\hline & {$[22](N=2)$} & 2.2022 & 2.3912 & 2.9578 & 3.6384 & 4.3736 & 5.1463 \\
\hline & Theorem $1(N=2)$ & 2.3367 & 2.5627 & 3.1084 & 3.7402 & 4.4340 & 5.1703 \\
\hline & Theorem $1(N=3)$ & 2.3485 & 2.5829 & 3.1371 & 3.7770 & 4.4779 & 5.2207 \\
\hline & Theorem $1(N=4)$ & 2.3526 & 2.5898 & 3.1469 & 3.7895 & 4.4928 & 5.2377 \\
\hline \multirow{8}{*}{0.9} & {$[3]$} & 1.3789 & 1.7424 & 2.4328 & 3.2234 & 4.0643 & - \\
\hline & {$[14]$} & 1.5304 & 1.8737 & 2.5048 & 3.2591 & 4.0744 & - \\
\hline & {$[16]$} & 1.6654 & 2.1251 & 2.7113 & 3.3839 & 4.1136 & - \\
\hline & {$[21]$} & 1.7157 & 2.2302 & 2.8630 & 3.5729 & 4.3343 & 5.1306 \\
\hline & {$[22](N=2)$} & 1.8828 & 2.3585 & 2.9578 & 3.6384 & 4.3736 & 5.1463 \\
\hline & Theorem $1(N=2)$ & 2.1377 & 2.5627 & 3.1084 & 3.7402 & 4.4340 & 5.1703 \\
\hline & Theorem $1(N=3)$ & 2.1486 & 2.5829 & 3.1371 & 3.7770 & 4.4779 & 5.2207 \\
\hline & Theorem $1(N=4)$ & 2.1524 & 2.5898 & 3.1469 & 3.7895 & 4.4928 & 5.2377 \\
\hline
\end{tabular}

where

\begin{tabular}{|c|c|c|c|c|c|c|c|}
\hline$\mu$ & Method & $h_{1}=0$ & $h_{1}=1$ & $h_{1}=2$ & $h_{1}=3$ & $h_{1}=4$ & $h_{1}=5$ \\
\hline \multirow{10}{*}{ Any $\mu$} & {$[3]$} & 1.3454 & 1.7424 & 2.4328 & 3.2234 & 4.0643 & - \\
\hline & {$[14]$} & 1.5296 & 1.8737 & 2.5049 & 3.2591 & 4.0744 & - \\
\hline & {$[16]$} & 1.6654 & 2.1251 & 2.7113 & 3.3839 & 4.1136 & - \\
\hline & [19] (Corollary 2) & 1.868 & 2.120 & 2.724 & 3.458 & 4.257 & 5.097 \\
\hline & {$[20]\left(\right.$ Theorem $\left.1 N_{v}=4\right)$} & 2.11 & 2.20 & 2.65 & - & - & - \\
\hline & {$[21]$} & 1.7157 & 2.2302 & 2.8630 & 3.5729 & 4.3343 & 5.1306 \\
\hline & {$[22](N=2)$} & 1.8828 & 2.3585 & 2.9578 & 3.6384 & 4.3736 & 5.1463 \\
\hline & Theorem $1(N=2)$ & 2.1377 & 2.5627 & 3.1084 & 3.7402 & 4.4340 & 5.1703 \\
\hline & Theorem $1(N=3)$ & 2.1486 & 2.5829 & 3.1371 & 3.7770 & 4.4779 & 5.2207 \\
\hline & Theorem $1(N=4)$ & 2.1524 & 2.5898 & 3.1469 & 3.7895 & 4.4928 & 5.2377 \\
\hline
\end{tabular}

$$
\left|\delta_{1}\right| \leq 1.6,\left|\delta_{2}\right| \leq 0.05,\left|\delta_{3}\right| \leq 0.1,\left|\delta_{4}\right| \leq 0.3 .
$$

Table 1 Maximum allowable delay bound $h_{2}$ for given $h_{1}$ and $\mu$

Table 2 Maximum allowable delay bound $h_{2}$ for given $h_{1}$ and unknown $\mu$ 
Table 3 Upper delay bound $h_{2}$ for given $h_{1}$

\begin{tabular}{|c|c|c|c|c|c|c|}
\hline \multirow{2}{*}{ Method } & \multicolumn{6}{|c|}{$h_{1}$} \\
\hline & 0 & 0.2 & 0.4 & 0.6 & 0.8 & 1.0 \\
\hline$[13]$ & 0.9442 & 0.9757 & 1.0208 & 1.0795 & 1.1500 & 1.2308 \\
\hline$[21]$ & 1.0571 & 1.0953 & 1.1385 & 1.1865 & 1.2392 & 1.2966 \\
\hline$[22](N=2)$ & 1.1030 & 1.1337 & 1.1703 & 1.2123 & 1.2594 & 1.3111 \\
\hline Theorem $2(N=2)$ & 1.1510 & 1.1783 & 1.2123 & 1.2527 & 1.2993 & 1.3515 \\
\hline Theorem $2(N=3)$ & 1.1557 & 1.1851 & 1.2214 & 1.2645 & 1.3140 & 1.3693 \\
\hline Theorem $2(N=4)$ & 1.1573 & 1.1875 & 1.2246 & 1.2686 & 1.3191 & 1.3755 \\
\hline
\end{tabular}

When there is no restriction on the delay derivative, Table 3 shows the obtained maximum allowable delay bound for a varying $h_{1}$. From the table, it is clear to see that for this example, some existing results have been improved.

Example $3^{[16]}$. Consider another uncertain system described by the matrices

$$
\begin{aligned}
& A=\left[\begin{array}{cc}
-0.5 & -2 \\
1 & -1
\end{array}\right], \quad B=\left[\begin{array}{cc}
-0.5 & -1 \\
0 & 0.6
\end{array}\right], \\
& D=\left[\begin{array}{ll}
1 & 0 \\
0 & 1
\end{array}\right], \quad E_{a}=\left[\begin{array}{cc}
0.2 & 0 \\
0 & 0.2
\end{array}\right], \\
& E_{b}=\left[\begin{array}{cc}
0.2 & 0 \\
0 & 0.2
\end{array}\right] .
\end{aligned}
$$

When $h_{1}=0$, Table 4 lists the maximum allowable delay bounds for different values of the delay derivative. It is found that the maximum allowable upper bounds of time-delay are $0.5594,0.5599,0.5601$ respectively when $N=2,3,4$, which are larger than those in $[16,21,22]$. Obviously, our criterion leads to much less conservative results.

Table 4 Maximum allowable delay bound $h_{2}$ for $h_{1}=0$

\begin{tabular}{cccc}
\hline \multirow{2}{*}{ Method } & \multicolumn{3}{c}{$\mu$} \\
\cline { 2 - 4 } & 0.5 & 0.9 & Any $\mu$ \\
\hline$[16]$ & 0.4760 & 0.4760 & 0.4760 \\
{$[21]$} & 0.4783 & 0.4783 & 0.4783 \\
{$[22](N=2)$} & 0.5151 & 0.5151 & 0.5151 \\
Theorem 2 $(N=2)$ & 0.5594 & 0.5594 & 0.5594 \\
Theorem 2 $(N=3)$ & 0.5599 & 0.5599 & 0.5599 \\
Theorem 2 $(N=4)$ & 0.5601 & 0.5601 & 0.5601 \\
\hline
\end{tabular}

\section{Conclusions}

This paper proposes a new approach for delay-dependent robust stability analysis of uncertain system with interval time-varying delay. The key features of the approach include delay decomposition technique for designing the L-K functional and a tighter integral inequality for bounding the cross-terms. As a result, less conservative results are achieved. Numerical examples have illustrated the effectiveness of the proposed method.

\section{Acknowledgement}

The authors would like to thank the associate editor, the anonymous reviewers and K. Ramakrishnan from Indian Institute of Technology Kharagpur for their insightful and constructive comments, which helped to enrich the content and improve the presentation of this paper.

\section{References}

[1] K. Q. Gu, V. L. Kharitonov, J. Chen. Stability of Timedelay Systems, Basel: Birkhauser, pp. 1-17, 2003.

[2] M. Wu, Y. He, J. H. She, G. P. Liu. Delay-dependent criteria for robust stability of time-varying delay systems. $A u-$ tomatica, vol. 40, no. 8, pp. 1435-1439, 2004.

[3] Y. He, Q. G. Wang, C. Lin, M. Wu. Delay-range-dependent stability for systems with time-varying delay. Automatica, vol. 43, no. 2, pp. 371-376, 2007.

[4] P. Park, J. W. Ko. Stability and robust stability for systems with a time-varying delay. Automatica, vol. 43, no. 10, pp. 1855-1858, 2007.

[5] C. Jeong, P. Park, S. H. Kim. Improved approach to robust stability and $H_{\infty}$ performance analysis for systems with an interval time-varying delay. Applied Mathematics and Computation, vol.218, no. 21, pp. 10533-10541, 2012.

[6] K. Q. Gu. An integral inequality in the stability problem of time-delay systems. In Proceedings of the 39th IEEE Conference on Decision and Control, IEEE, Sydney, Australia, pp. 2805-2810, 2000.

[7] Q. L. Han. Absolute stability of time-delay systems with sector-bounded nonlinearity. Automatica, vol. 41, no. 12 , pp. 2171-2176, 2005.

[8] X. M. Zhang, Q. L. Han. New Lyapunov-krasovskii functionals for global asymptotic stability of delayed neural networks. IEEE Transactions on Neural Networks, vol. 20, no. 3, pp. 533-539, 2009.

[9] J. Sun, G. P. Liu, J. Chen, D. Rees. Improved delayrange-dependent stability criteria for linear systems with time-varying delays. Automatica, vol. 46, no. 2, pp. 466-470, 2010

[10] O. M. Kwon, J. H. Park, S. M. Lee. An improved delaydependent criterion for asymptotic stability of uncertain dynamic systems with time-varying delays. Journal of Optimization Theory and Applications, vol. 145, no. 2, pp. 343$353,2010$.

[11] K. Ramakrishnan, G. Ray. Delay-range-dependent stability criterion for interval time-delay systems with nonlinear perturbations. International Journal of Automation and Computing, vol. 8, no. 1, pp. 141-146, 2011. 
[12] W. Zhang, X. S. Cai, Z. Z. Han. Robust stability criteria for systems with interval time-varying delay and nonlinear perturbations. Journal of Computational and Applied Mathematics, vol. 234, no. 1, pp. 174-180, 2010.

[13] X. F. Jiang, Q. L. Han. New stability criteria for linear systems with interval time varying delay. Automatica, vol. 44, no. 10 , pp. 2680-2685, 2008.

[14] H. Y. Shao. New delay-dependent stability criteria for systems with interval delay. Automatica, vol. 45, no. 3, pp. 744$749,2009$.

[15] X. L. Zhu, Y. Wang, G. H. Yang. New stability criteria for continuous-time systems with interval time-varying delay. IET Control Theory and Applications, vol. 4, no. 6, pp. 1101-1107, 2010.

[16] C. Peng, Y. C. Tian. Improved delay-dependent robust stability criteria for uncertain systems with interval timevarying delay. IET Control Theory and Application, vol. 2, no. 9 , pp. $752-761,2008$.

[17] Q. L. Han. A delay decomposition approach to stability of linear neutral systems. In Proceedings of the 17th World Congress, The International Federation of Automatic Control, IEEE, Seoul, Korea, pp. 2607-2612, 2008.

[18] Q. L. Han. A discrete delay decomposition approach to stability of linear retarded and neutral systems. Automatica, vol. 45, no. 2, pp. 517-524, 2009.

[19] E. Fridman, U. Shaked, K. Liu. New conditions for delayderivative-dependent stability. Automatica, vol. 45, no. 11 pp. 2723-2727, 2009.

[20] C. Peng, M. R. Fei. A refined delay-partitioning approach to the stability of linear systems with interval time-varying delays. In Proceedings of the 2013 IEEE International Symposium on Industrial Electronics, IEEE, Taipei, China, pp. 1$5,2013$.

[21] K. Ramakrishnan, G. Ray. Delay-dependent robust stability criteria for linear uncertain systems with interval time varying delay. In Proceedings of IEEE Region 10 Conference, IEEE, Singapore, pp. 1-6, 2009.

[22] K. Ramakrishnan, G. Ray. Robust stability criteria for uncertain linear systems with interval time-varying delay. Journal of Control Theory and Applications, vol.9, no. 4, pp. 559-566, 2011.

[23] J. M. Jiao. Robust stability and stabilization of discrete singular systems with interval time-varying delay and linear fractional uncertainty. International Journal of Automation and Computing, vol. 9, no. 1, pp. 8-15, 2012.

[24] P. Balasubramaniam, T. Senthilkumar. Delay-dependent robust stabilization and $H_{\infty}$ control for uncertain stochastic T-S fuzzy systems with discrete interval and distributed time-varying delays. International Journal of Automation and Computing, vol. 10, no. 1, pp. 18-31, 2013.
[25] D. Yue, E. G. Tian, Y. J. Zhang. A piecewise analysis method to stability analysis of linear continuous/discrete systems with time-varying delay. International Journal Robust Nonlinear Control, vol. 19, no. 13, pp. 1493-1518, 2009.

[26] L. H. Xie. Output feedback control of systems with parameter uncertainty. International Journal of Control, vol.63, no. 4, pp. 741-750, 1996.

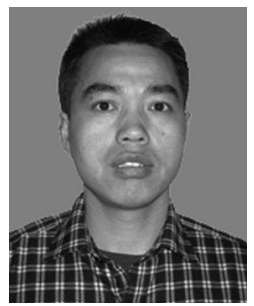

Jun-Jun Hui received his B.Sc. and M. Sc. degrees in control engineering from $\mathrm{Xi}^{\prime}$ an Research Institute of High Technology, China in 2001 and 2005, respectively. He is currently pursuing his $\mathrm{Ph}$. D. degree in control science and engineering at the 301 Section, $\mathrm{Xi}^{\prime}$ an Research Institute of High Technology.

His research interests include time-delay systems and networked control systems.

E-mail: ep22stone@163.com (Corresponding author) ORCID iD: 0000-0002-6220-8857

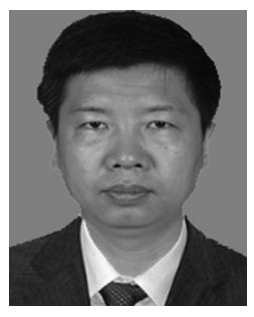

He-Xin Zhang received his M.Sc. degree in control science and engineering from Northwestern Polytechnical University, China in 1994, and his Ph.D. degree in control science and engineering from $\mathrm{Xi}^{\prime}$ an Research Institute of High Technology. Now he works as a professor in the 301 Section, Xi'an Research Institute of High Technology, China.

His research interests include nonlinear system identification and control, measurement and control, and adaptive control.

E-mail: epzhx301@163.com

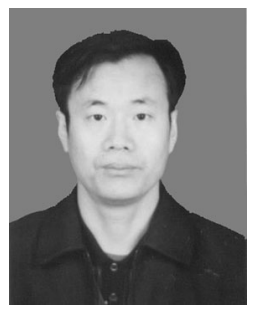

Xiang-Yu Kong received his B. Sc. degree in control science and engineering from Beijing Institute of Technology, China in 1990, his M. Sc. degree in control science and engineering from $\mathrm{Xi}^{\prime}$ an Research Institute of High Technology, China in 2000, and his Ph. D. degree in control science and engineering from $\mathrm{Xi}^{\prime}$ an Jiaotong University, China in 2005, respectively. Now he works as a professor in $\mathrm{Xi}^{\prime}$ an Research Institute of High Technology, China.

His research interests include adaptive signal processing, nonlinear system modeling and its applications, and fault diagnosis. E-mail: xiangyukong01@163.com

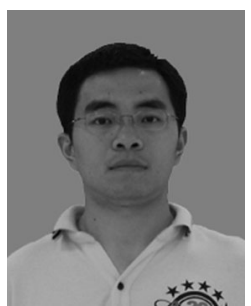

Xin Zhou received his B. Sc. degree in control science and engineering from Northeastern University, China in 2002, and his M. Sc. degree in control science and engineering from National University of Defense Technology, China in 2004. Now he works as a lecturer in the 301 Section, $\mathrm{Xi}^{\prime}$ an Research Institute of High Technology, China

His research interests include nonlinear system, time-delay systems and networked control systems.

E-mail: ascentor@163.com 